\title{
Morfoanatomía e histoquímica de cuatro Asteraceae nativas del Bosque Chaqueño Serrano (Córdoba, Argentina)
}

\author{
NATALÍ TOSORATTO1 ${ }^{1}$ MARÍA TERESA COSA ${ }^{1}$ y NATALIA DELBÓN¹
}

\begin{abstract}
Summary: Morfoanatomy and histochemistry in Asteraceae native from Chaco Serrano Forest (Córdoba, Argentina). Chaco Serrano Forest is characterized by a semiarid climate; the species that live there have modifications related mainly to water balance. In this habitat the Asteraceae family is one of the largest, concentrating large numbers of species with medicinal properties. Four of them were studied in this work, they are: Austroeupatorium inulifolium, Baccharis salicifolia, Chromolaena hookeriana and Ophryosporus axilliflorus. We inquired about air vegetative organs in order to understand the adaptive strategies presented in relation to the environment in which they live, detect active producing structures and infer the chemicals they produce. In the four studied species xeromorphic features were observed, including the presence of trichomes, ducts and endodermis with Caspary band in the stem. Also they were positive in pectic and lipid compounds test and $B$. salicifolia presented tannins in the epidermis. We conclude that the secretory structures and chemicals found were directly related to the medicinal properties of these species.
\end{abstract}

Key words: Austroeupatorium inulifolium, Baccharis salicifolia, Chromolaena hookeriana, Ophryosporus axilliflorus, secretory duct, trichomes, endodermis, Caspary band.

Resumen: El Bosque Chaqueño Serrano se caracteriza por un clima semiárido; las especies que habitan en él presentan una serie de modificaciones relacionadas principalmente al balance hídrico. En este hábitat la familia Asteraceae es una de las más numerosas, nucleando gran cantidad de especies con propiedades medicinales. Cuatro de ellas fueron estudiadas en el presente trabajo, ellas son: Austroeupatorium inulifolium, Baccharis salicifolia, Chromolaena hookeriana y Ophryosporus axilliflorus. Se indagó sobre los órganos vegetativos aéreos con el objetivo de comprender las estrategias adaptativas que presentan en relación al ambiente en que viven, detectar estructuras productoras de principios activos e inferir los compuestos químicos que ellas producen. En las cuatro especies estudiadas se observaron características consideradas xeromórficas, destacándose la presencia de tricomas, conductos y endodermis con banda de Caspary en los tallos. Además, resultaron positivas para las pruebas de compuestos pécticos y lipídicos y $B$. salicifolia presentó taninos en la epidermis. Concluimos que las estructuras secretoras y los compuestos químicos encontrados estarían directamente relacionados con las propiedades medicinales de estas especies.

Palabras clave: Austroeupatorium inulifolium, Baccharis salicifolia, Chromolaena hookeriana, Ophryosporus axilliflorus, conducto secretor, tricoma, endodermis, banda de Caspary.

1 Laboratorio de Morfología Vegetal. Instituto Multidisciplinario de Biología Vegetal (UNC-CONICET). Facultad de Ciencias Exactas, Físicas y Naturales. Universidad Nacional de Córdoba. 


\section{INTRODUCCIÓN}

El Bosque Chaqueño Serrano presenta suelos pobres en nutrientes, escasas precipitaciones, altas temperaturas en verano y una gran exposición a la radiación solar (Giorgis et al., 2011). En esta zona, las especies vegetales presentan una serie de modificaciones estructurales, anatómicas y fisiológicas, relacionadas principalmente con el transporte y almacenamiento de agua y para evitar su pérdida por transpiración (Fahn \& Cutler, 1992; Delbón et al., 2010; 2012; Giorgis et al., 2011). Además, una gran cantidad de especies poseen importancia farmacobotánica y son utilizadas en medicina popular; solo en la provincia de Córdoba se reconocen un total 669 taxones con propiedades medicinales, de los cuales la mayor proporción pertenece a la familia Asteraceae (Barboza et al., 2006).

A pesar de la importancia que poseen los bosques serranos, en las últimas décadas se ha observado una gran pérdida de áreas naturales y un marcado deterioro de la vegetación, producto de la actividad antrópica (Gurvich et al., 2005; Hoyos et al., 2010; Giorgis et al., 2013). Por lo tanto, el estudio de las especies nativas y endémicas merece especial atención, por su importancia ecológica y su potencial interés económico.

En este marco, se estudiaron cuatro especies arbustivas de Asteraceae, nativas de la zona serrana de la provincia de Córdoba, Austroeupatorium inulifolium, Baccharis salicifolia, Chromolaena hookeriana y Ophryosporus axilliflorus, a las cuales se le atribuyen propiedades medicinales (Nuñez \& Cantero, 2000; Barboza et al., 2006). A. inulifolium se utiliza como anticonceptivo, emenagogo y abortivo, B. salicifolia presenta actividad antileishmaniasis, insecticida, antimicrobiana, antirreumática, antiinflamatoria, antiséptica y astringente, en tanto que $O$. axilliflorus es analgésica, antisifilítica, astringente y dermopática. En general, estas especies presentan compuestos químicos entre los cuales se destacan flavonoides, terpenos, lactonas y cromonas, (Jakupovic et al., 1990; Favier et al., 1998; Freire \& Urtubey, 1999; Nuñez \& Cantero, 2000). A pesar de su potencial farmacobotánico, no existen investigaciones anatómicas o histoquímicas de estas especies. Por ello, en este trabajo se estudian los órganos vegetativos aéreos con el objetivo de comprender las estrategias adaptativas que presentan en relación al ambiente en que viven, detectar estructuras productoras de principios activos e inferir los compuestos químicos que ellas producen.

\section{Materiales y Métodos}

Las especies analizadas proceden de la Reserva Hídrica Natural Parque La Quebrada, Córdoba. Depto. Colón, Prov. Córdoba, Argentina. El material de herbario correspondiente se encuentra depositado en el Museo Botánico de Córdoba (CORD).

Austroeupatorium inulifolium (Kunth) R. M. King \& H. Rob. (COSA 401, 19/05/2013),

Baccharis salicifolia (Ruiz \& Pav.) Pers. (COSA 403, 19/05/2013),

Chromolaena hookeriana (Griseb.) R. M. King \& H. Rob. (COSA 402, 19/05/2013),

Ophryosporus axilliflorus (Griseb.) Hieron. (COSA 400, 19/05/2013).

De cada población se eligieron al azar cuatro individuos adultos y sanos (4 réplicas) y se tomaron muestras que fueron fijadas en FAA. Para realizar los estudios foliares se tomaron de cada individuo cinco hojas maduras y sanas (5 pseudoréplicas), ubicadas en la zona apical de las ramas laterales, entre el tercer y quinto nudo. El estudio del tallo con crecimiento primario fue realizado en el primer y segundo entrenudo inmediatamente por debajo de la yema apical, en tanto que el estudio del tallo con crecimiento secundario se realizó entre el tercero y sexto nudo.

Se realizaron extendidos de ambas caras foliares utilizando la técnica de "peeling" (D' Ambrogio de Argüeso, 1986). En cada uno se seleccionaron al azar cinco campos en los que se determinó el número de células epidérmicas, estomas, tricomas glandulares y eglandulares. Se calculó el índice estomático con la fórmula (Stace, 1965): I.E.= (frecuencia de estomas $/$ frecuencia de estomas + frecuencia de células epidérmicas) X 100. El estudio de los tricomas se realizó siguiendo la clasificación y terminología propuesta por Ramayya (1962) para la familia Asteraceae.

Para las observaciones anatómicas, se realizaron preparados permanentes de cortes transversales de lámina foliar. El material prefijado fue incluido 


\section{N. Tosoratto et al. - Morfoanatomía e histoquímica de cuatro Asteraceae}

en histoplast por la metodología convencional (D'Ambrogio de Argüeso, 1986), cortado con micrótomo de rotación $(10 \mu \mathrm{m})$, coloreado con azul astral-fucsina básica y montado con bálsamo de Canadá (Kraus et al., 1998). Además, se realizaron preparados temporarios de cortes transversales a mano alzada por lámina, pecíolo, tallo con crecimiento primario y secundario, los cuales se tiñeron con azul astral-fucsina básica y se montaron con glicerina $50 \%$.

Para las mediciones foliares se realizaron preparados temporarios de cortes transversales a mano alzada por lámina prefijadas. Se utilizaron 5 hojas por individuo (5 pseudoréplicas), y se confeccionaron 5 cortes por cada una, en ellas se midió el espesor total de la lámina, del parénquima en empalizada y del esponjoso. Se calculó la relación esponjoso/ empalizada como la fórmula, Es/Em= Espesor parénquima esponjoso $(\mathrm{mm}) /$ Espesor parénquima empalizada $(\mathrm{mm})$.

Por último, cortes a mano alzada de hoja, pecíolo y tallo fijadas en FAA fueron sometidos a pruebas histoquímicas (Johansen, 1940). Se empleó Sudan IV para detectar la presencia de lípidos, Rojo de Rutenio para compuestos pécticos, Sulfato de Cobre para resinas, Cloruro Férrico al $10 \%$ y Carbonato de Sodio para compuestos fenólicos (taninos) y la tinción de Draguendorf para alcaloides.

Todas las variables se analizaron empleando el programa InfoStat (Di Rienzo et al., 2011), calculándose la media y desvío estándar de cada variable. Para analizar las características epidérmicas se realizó, en primer lugar, Análisis de Varianza con el objetivo de determinar si existen diferencias significativas entre el epifilo e hipofilo de una misma especie; luego, se realizaron tests de Tukey para comprobar las diferencias entre las especies, teniendo en cuenta las caras abaxiales por un lado y las adaxiales por otro $(n=100, p>0,05)$. Las mediciones foliares se compararon entre especies utilizando tests de Tukey $(n=60, p>0,05)$.

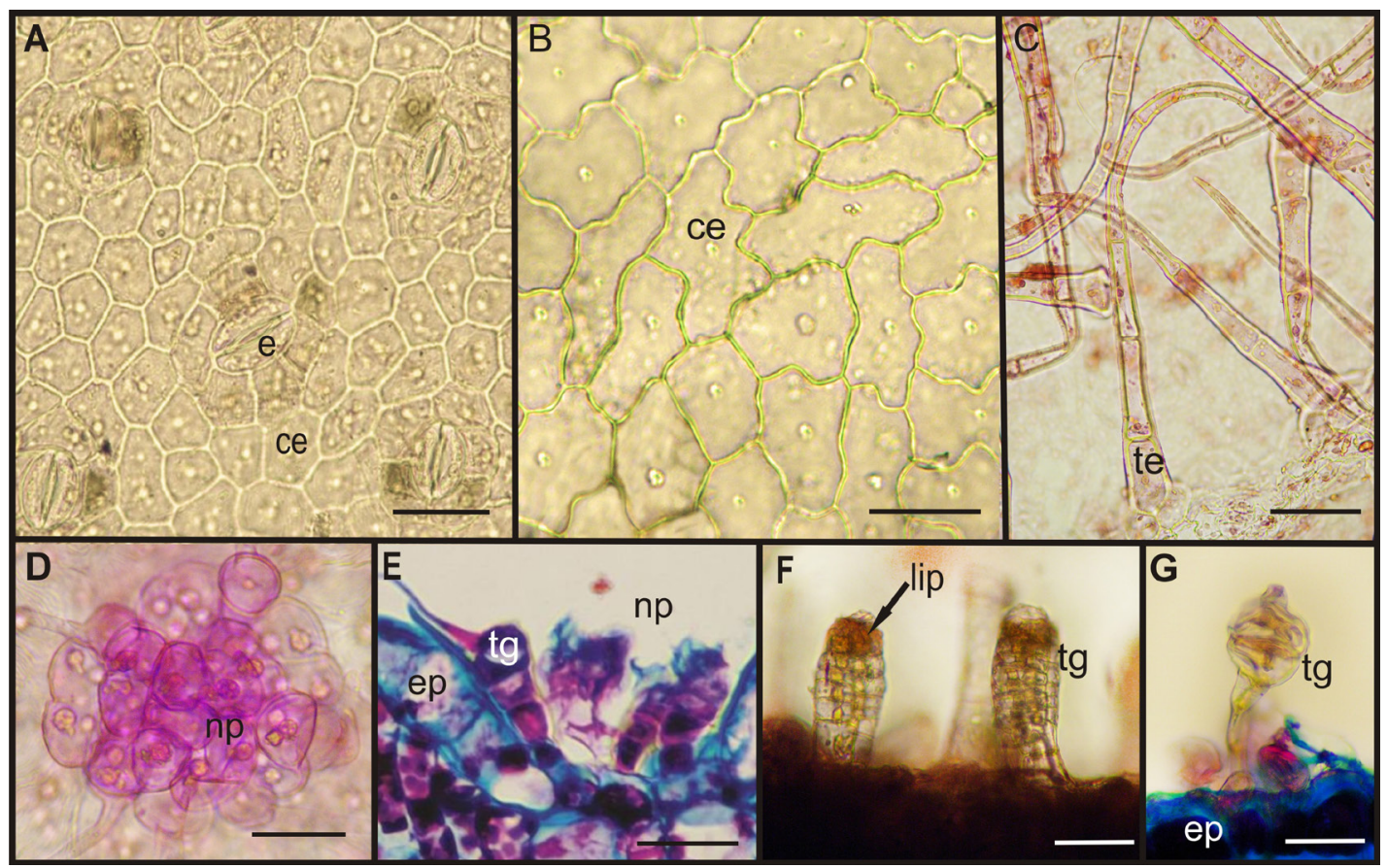

Fig. 1. Epidermis foliar. A-D: Vista superficial. E-G: Corte transversal. A: Hipofilo con estomas anomocíticos. B: Epifilo sin estomas. C: Tricomas cónico simple. D: Nido piloso. E: Tricomas bulbífero flagelado con célula apical y bicelular biseriado en nido piloso. F: Tricomas glandular biseriado con lípidos. G: Tricoma filiforme capitado. A, D y E: B. salicifolia. B-C: O. axilliflorus. F: C. hookeriana. G: A. inulifolium. Abreviaturas: ce: célula epidérmica propiamente dicha, e: estomas, ep: epidermis, $\mathrm{np}$ : nido piloso, lip: lípidos, tg: tricoma glandular, te: tricoma eglandular. Escalas: A-C, F-G: $25 \mu \mathrm{m}, \mathrm{D}-\mathrm{E}: 50 \mu \mathrm{m}$. 


\section{Resultados}

Hoja

Vista superficial

Las cuatro especies presentaron estomas en ambas caras foliares a excepción de $O$. axilliflorus que resultó hipostomática (Fig. $1 \mathrm{~A}, \mathrm{~B}$ ). Todas las especies presentaron estomas anomocíticos. En $B$. salicifolia se hallaban rodeados por 4 a 7 células epidérmicas propiamente dichas (Fig. 1 A), en tanto que en las restantes especies por 4 a 5 células.

Fueron determinados 4 tipos de tricomas en las diferentes especies, 1 eglandular y 3 glandulares:

Eglandular cónico simple: pie unicelular y cuerpo pluricelular uniseriado, formado por 7 células de paredes gruesas, las terminales alargadas, pudiendo desprenderse a la madurez. Se observaron en ambas caras foliares, pecíolo y tallo de $A$. inulifolium, C. hookeriana y O. axilliflorus (Fig. 1 C), siendo ausente en $B$. salicifolia.

2- Glandular Bulbíferos flagelados con célula apical: pie pluricelular, uniseriado, de 3 a 4 células y cabezuela unicelular globosa, con una célula apical flageliforme. Se observaron agrupados, formando nidos pilosos en ambas caras foliares y en el pecíolo de B. salicifolia (Fig. 1 D, E)

3- Glandular biseriado: pie bicelular biseriado y cuerpo biseriado de 3 a 6 células. En $C$. hookeriana se ubicaron en el hipofilo y pecíolo (Fig. $1 \mathrm{~F}$ ), en tanto que en $B$. salicifolia presentan cabeza globosa $\mathrm{y}$ forman los nidos pilosos (Fig. $1 \mathrm{E}$ ).

5- Glandular Filiforme Capitado: pie pluricelular, uniseriado, formado por 3 a 5 células cilíndricas y una cabezuela de 1 a 2 células de forma ovoide. Se presentaron solamente en el hipofilo de $A$. inulifolium (Fig. $1 \mathrm{G})$.

Todas las variables epidérmicas analizadas mostraron diferencias significativas entre las caras foliares de cada especie, excepto para la frecuencia estomática y de tricomas eglandulares de $A$. inulifolium. En todas las especies se observó una mayor frecuencia de estomas en el hipofilo siendo B. salicifolia y C. hookeriana las que presentaron mayores valores. Los tricomas eglandulares fueron más abundantes en el hipofilo para todas las especies, excepto $B$. salicifolia que no presentó este tipo de tricomas. Los glandulares fueron muy abundantes en $C$. hookeriana, en menor proporción en $A$. inulifolium, ausentes en $O$. axilliflorus y formando nidos pilosos en B. salicifolia (Tabla 1).

\section{Corte transversal de lámina y peciolo}

Las cuatro especies mostraron una epidermis uniestratificada con cutícula fina (Fig. 2 A-D), y estomas al mismo nivel o ligeramente por encima de las células epidérmicas (Fig. 2 A, G). El sistema vascular estuvo representado por hacecillos colaterales cerrados de diferentes tamaños, rodeados por una vaina parenquimática (Fig. 2 A-D), pudiendo estar acompañados de conductos secretores de origen esquizógenos de epitelio secretor uniestratificado (Fig. 2 B, E).

Las diferencias más importantes entre las especies se observaron en las características del mesófilo y en el número de haces vascular en vena media de la lámina y en pecíolo. Baccharis salicifolia presentó hojas con mayor espesor total, mesófilo con estructura isolateral y mayor relación entre el parénquima empalizada y el esponjoso, valor superior a 1 (Fig. 2 A, Tabla 1). Las restantes especies presentaron estructura dorsiventral, de ellas O. axilliflorus mostró la mayor relación empalizada esponjoso y fue la especie con menor espesor foliar (Tabla 1). Con respecto al sistema de conducción, B. salicifolia y A. inulifolium presentaron 1 haz central en vena media (Fig. 2 C), mientras que, las restantes presentaron 3 (Fig. 2 D). En el peciolo, A. inulifolium presentó 3 hacecillos (Fig. 2 E), B. salicifolia 5 , en tanto que $C$. hookeriana y O. axilliflorus mostraron 7.

\section{Tallo}

\section{Corte transversal}

En A. inulifolium, C. hookeriana y O. axilliflorus los tallos fueron circulares en sección transversal. En todas ellas la epidermis fue unistratificada, con cutícula engrosada y ticomas eglandulares cónicos. La corteza estuvo formada por unos pocos estratos de colénquima angular y clorénquima (Fig. $3 \mathrm{~A}$ ).

En B. salicifolia, el tallo presentó forma irregular en sección transversal, con numerosas costillas, las que se correspondieron con haces vasculares de mayores dimensiones. En las costillas, la epidermis uniestratificada presentó mayor espesor y su cutícula fue estriada, por debajo se encontraron numerosas capas de colénquima angular. Entre las costillas, la epidermis fue más fina y por debajo se observó clorénquima (Fig. 3 B).

En todas las especies el tejido vascular formó una eustela con hacecillos colaterales abiertos, acompañados de fibras (Fig. 3 A y B). Rodeando 


\section{N. Tosoratto et al. - Morfoanatomía e histoquímica de cuatro Asteraceae}

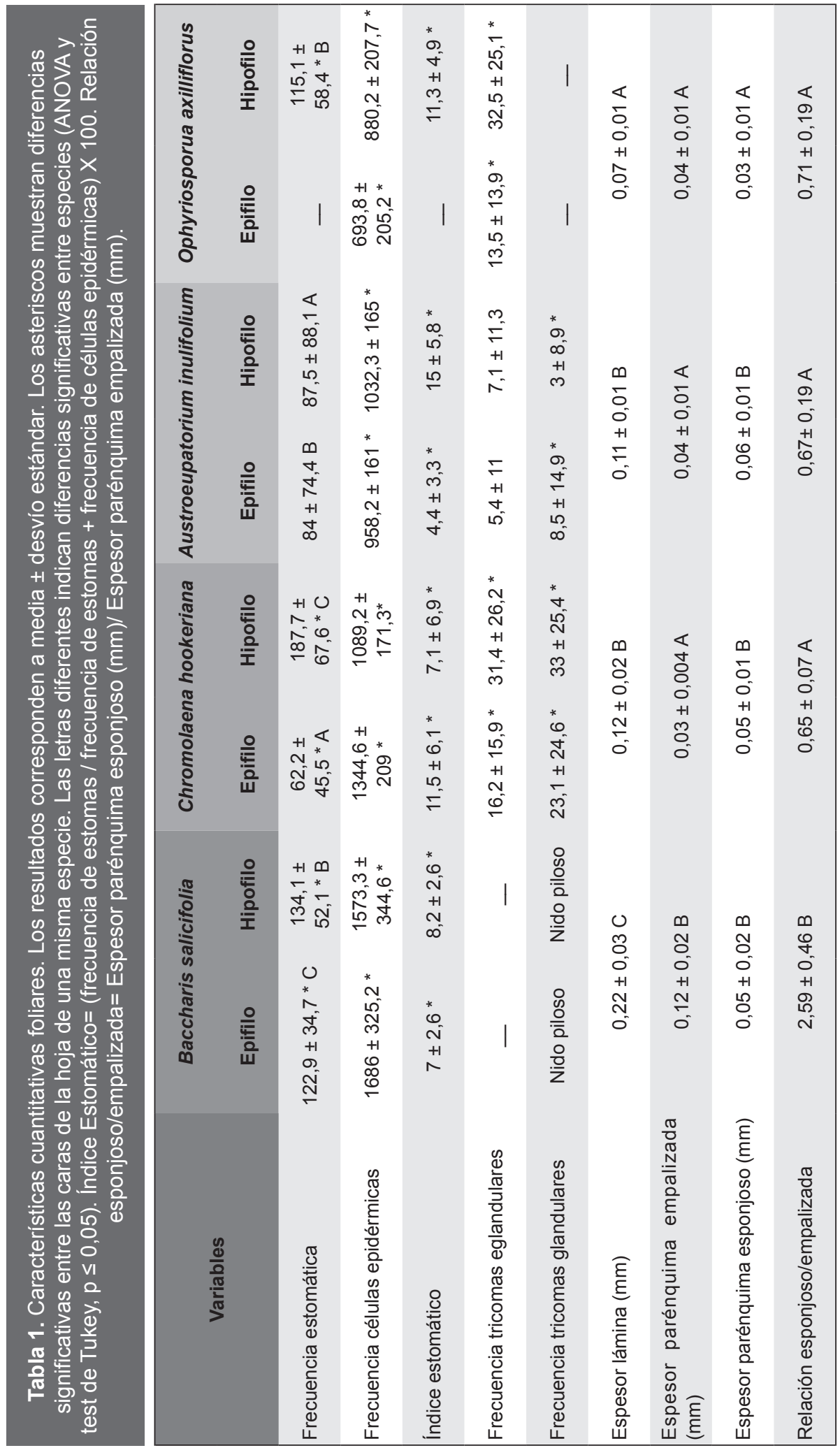




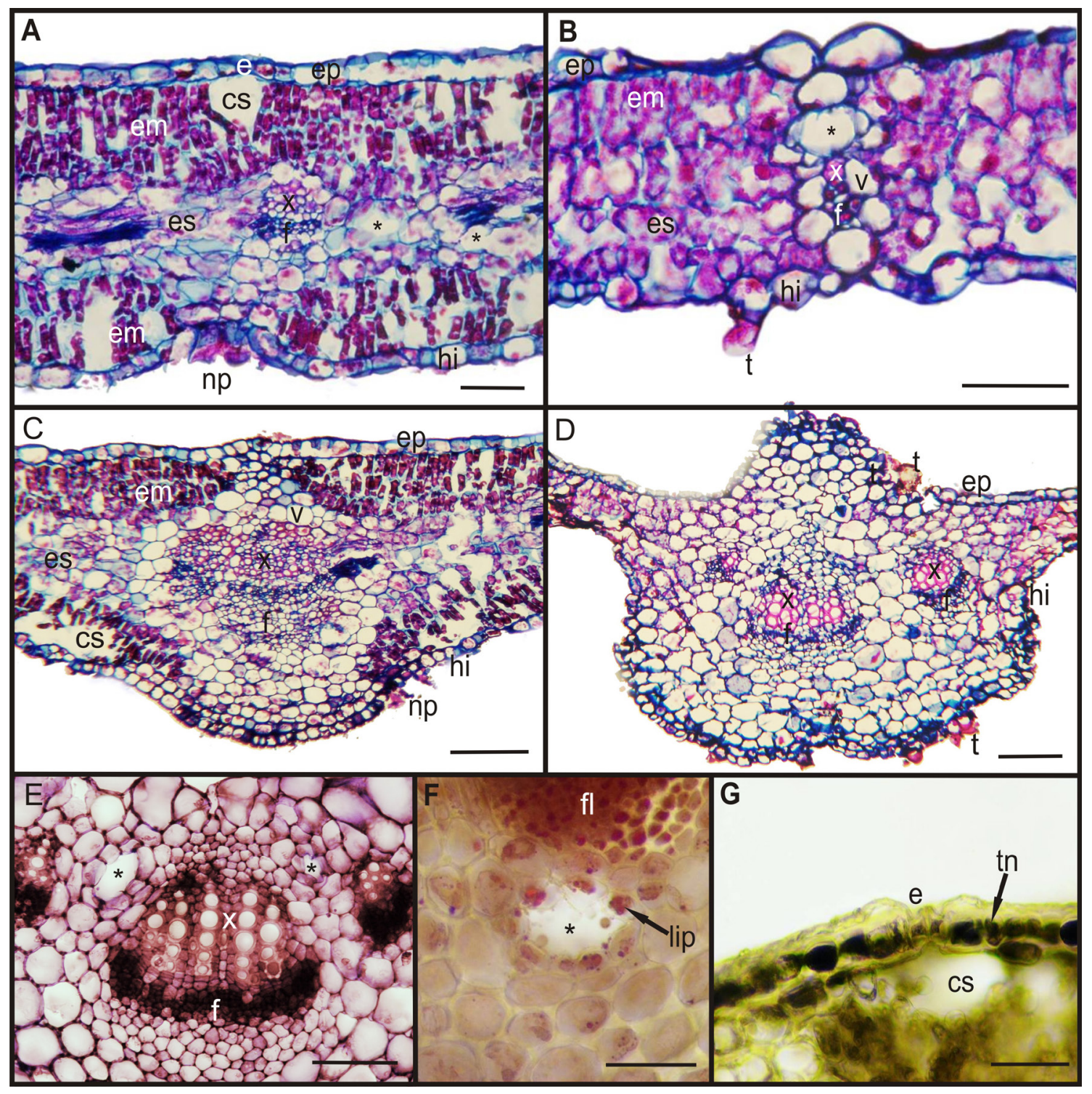

Fig. 2. Hoja en corte transversal. A-B, G: Lámina. C-D: Vena Media. E-F: Pecíolo. A: Mesofilo isolateral. B: Mesofilo dorsiventral. C: Vena media con un haz central. D: Vena media con tres haces. E: Pecíolo con tres haces vasculares y conductos secretores. F: Lípidos en conductos. G: Taninos en epidermis. A, C y G: $B$. salicifolia. B y D: C. hookeriana. E y F: A. inulifolium. Abreviaturas: cs: cámara subestomática, e: estoma, em: parénquima en empalizada, ep: epifilo, es: parénquima esponjoso, f: floema, hi: hipofilo, lip: lípidos, $\mathrm{np}$ : nido piloso, t: tricoma, tn: taninos, v: vaina parenquimática, $\mathrm{x}$ : xilema, asterisco: conductos secretores. Escalas: C-D: $100 \mu \mathrm{m}, \mathrm{A}-\mathrm{B}, \mathrm{E}: 50 \mu \mathrm{m}, \mathrm{F}-\mathrm{G}: 20 \mu \mathrm{m}$.

el cilindro central se observó una endodermis continua, formada por un estrato de células (Fig. 3 A-C). Por su parte, se observaron canales secretores en la corteza de todas las especies (Fig. 3 A y B), pero solo en la médula de $A$. inulifolium
(Fig. 3 A).

El crecimiento secundario comenzó con el desarrollo de la peridermis, que rápidamente reemplazó a la epidermis (Fig. 3 D), excepto en los tallos de $A$. inulifolium en los que su desarrollo fue 


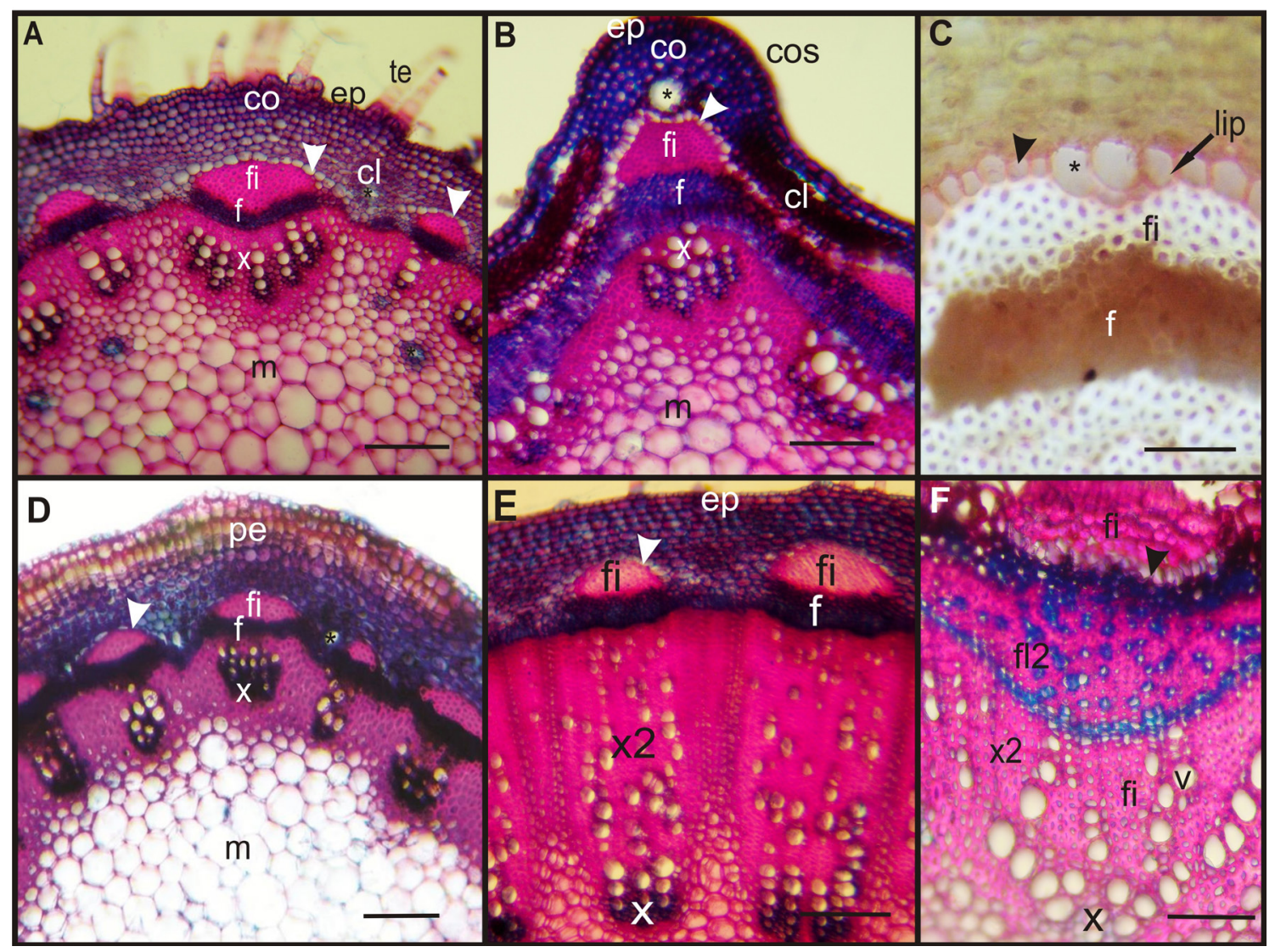

Fig. 3. Tallo en corte transversal. A-C: Tallo con crecimiento primario. D-F: Tallo con crecimiento secundario. A: Tallo joven con contorno circular. B: Tallo con costilla. C: Endodermis con presencia de lípidos formando Banda de Caspary. D: Comienzo de formación de peridermis. E: Desarrollo de xilema secundario. F: Detalle de xilema y floema secundarios. A y E: A. inulifolium. B y F: B. salicifolia. C y D: O. axilliflorus. Abreviaturas: cos: costilla, ep: epidermis, fi: fibras, f: floema, f2: floema secundario, líp: lípidos, m: médula, pe: peridermis, te: tricoma eglandular, $v$ : vasos, $x$ : xilema, $x 2$ : xilema secundario, asterisco: conductos secretores, punta de flecha: endodermis. Escalas: A-B, D-E: $100 \mu \mathrm{m}, \mathrm{C}, \mathrm{F}: 50 \mu \mathrm{m}$.

tardío (Fig. 3 E). El cambium formó normalmente floema centrífugamente y xilema centrípetamente (Fig. 3 E). El floema secundario de $B$. salicifolia se diferenció de los demás por estar formado en mayor proporción por fibras, con pequeños grupos de elementos conductores y células parenquimáticas dispersos entre ellas (Fig. 3 F).

\section{Pruebas histoquímicas}

Las cuatro especies resultaron positivas para las pruebas de compuestos pécticos, lipídicos y taninos; mientras que, fueron negativas para alcaloides y resinas.
Los lípidos fueron revelados en todas las especies en todos los órganos analizados, ubicados en los conductos secretores y en células parenquimáticas de la corteza (Fig. 2 F). Además, se observaron en la endodermis de $C$. hookeriana y $O$. axilliflorus, formando la banda de Caspary (Fig. 3 C); y en los tres tipos de tricomas glandulares (Fig. 1 F).

Los compuestos pécticos se evidenciaron en vasos, fibras, colénquima y peridermis; en tanto que $B$. salicifolia fue la única especie que resultó positiva para taninos, los cuales fueron detectados en la epidermis de la hoja, pecíolo, peridermis y tallo (Fig. 2 G). 


\section{Discusión}

En general, las especies que habitan ambientes áridos y semiáridos presentan adaptaciones relacionadas al balance hídrico, las cuales incluyen modificaciones en el área foliar, densidad de estomas, espesor del parénquima en empalizada, proporción de fibras, entre otros (Fahn \& Cutler, 1992; Delbón et al., 2010, 2012). Las especies estudiadas resultaron anfiestomáticas, excepto $\mathrm{O}$. axilliflorus, y presentaron abundantes estomas, siendo $B$. salicifolia la que mostró mayor frecuencia. Ambas características favorecerían el intercambio gaseoso y aumentarían la tasa fotosintética (Fahn \& Cutler, 1992). El mesófilo presentó estructura dorsiventral, excepto en $B$. salicifolia que fue isolateral, en esta especie fue mayor la relación entre parénquima empalizada y esponjoso, con lo cual sería mayor la actividad fotosintética. Nuestras observaciones concuerdan con lo reportado para otras especies de Baccharis (Budel \& Duarte, 2004; 2007, Molares et al., 2009) y Eupatorium (Cordati \& Gattuso, 1994).

En los tallos con crecimiento primario y secundario se destacan abundantes fibras y la presencia de endodermis. Ambas características se consideran adaptaciones a ambientes xéricos y su función sería la de proteger los tejidos vasculares y prevenir la desecación (Fahn \& Cutler, 1992). En general, la presencia de endodermis en los tallos es poco frecuente, aunque en la familia Asteraceae existen registros de este tejido con diversas características. Así, para Baccharis se ha reportado como una capa uniseriada continua o discontinua, con o sin banda de Caspary (Ariza Espinar, 1973; Budel \& Duarte, 2007); en las cuatro especies estudiadas, la endodermis resultó uniseriada continua y en $C$. hookeriana y $O$. axilliflorus presentó bandas de Caspary.

En Asteraceae es común la presencia de estructuras secretoras, productoras de numerosos compuestos químicos; estas le confieren a la familia un gran valor fitoquímico, pero además pueden ser consideradas como adaptaciones xeromórficas (Fahn \& Cutler, 1992; Delbón et al., 2012). En las especies estudiadas se han detectado tres tipos de tricomas glandulares y uno eglandulares; en tanto que en B. salicifolia, los tricomas se encuentran agrupados en nidos pilosos, siendo este carácter común en el género (Freire et al., 2007; Rodríguez et al., 2008; Pérez Cuadra \& Cambi, 2014). La presencia de tricomas ayudaría a disminuir la temperatura de las hojas y en consecuencia la pérdida de agua por transpiración (Fahn \& Cutler, 1992; Vilela et al., 2011; Delbón et al., 2012). Además, en todas las especies y en todos los órganos vegetativos se encontraron conductos secretores de origen esquizógeno, los cuales resultaron positivos solo para lípidos. Se consideran como una defensa contra los herbívoros y patógenos ya que su secreción solo saldría a la superficie si la planta es herida (Fahn \& Cutler, 1992; Delbón et al., 2012).

Con respecto a las pruebas histoquímicas, las cuatro especies dieron positivas para compuestos pécticos y lípidos, pero fueron negativas para alcaloides. Esta última prueba no coinciden con lo reportado anteriormente en $A$. inulifolium, ya que se reportaron alcaloides, así como aceites esenciales con derivados terpénicos, euparina y lactonas sesquiterpénicas (Cordatti \& Gatuso, 1994). Sumado a esto, en B. salicifolia se evidenciaron taninos en la epidermis, lo que le otorgaría por un lado, rigidez estructural, protección contra la deshidratación y descomposición; y por otro, sería importante en la defensa de las plantas contra herbívoros, ya que reducen la digestibilidad de los nutrientes (Feeny, 1970; Castro et al., 1999; Espírito-Santo, et al., 1999).

\section{Conclusiones}

En las cuatro especies estudiadas se observaron características consideradas xeromórficas y se destaca la presencia de tricomas y conductos secretores en todos los órganos y endodermis con Banda de Caspary en los tallos. Además, resultaron positivas para las pruebas de compuestos pécticos y lipídicos y $B$. salicifolia presentó taninos en la epidermis. Concluimos que las estructuras secretoras y compuestos químicos encontrados podrían estar relacionados con las propiedades medicinales de estas especies.

\section{Agradecimientos}

Agradecemos al Dr. Luis Ariza Espinar por la identificación de las especies y al financiamiento de de la Secretaría de Ciencia y Técnica, Universidad 


\section{N. Tosoratto et al. - Morfoanatomía e histoquímica de cuatro Asteraceae}

Nacional de Córdoba (SECYT-UNC) y del Consejo Nacional de Investigaciones Científicas y Técnicas (CONICET). ND es becaria posdoctoral de CONICET.

\section{Bibliografía}

ARIZA ESPINAR, L. A. 1973. Las especies de Baccharis (Compositae) de Argentina Central. Bol. Acad. Nac. Ci. 50: 176-305.

BARBOZA, G., J. J. CANTERO, C. NUÑEZ \& L. ARIZA ESPINAR. 2006. Flora medicinal de la Provincia de Córdoba (Argentina). Museo Botánico de Córdoba. Córdoba.

BUDEL, J. \& M. DUARTE. 2004. Morfoanatomia foliar y caulinar de Baccharis dracunculifolia DC, Asteraceae. Acta Farm. Bonaer. 23: 477-83.

BUDEL, J. \& M. DUARTE. 2007. Caracteres morfoanatômicos de partes vegetativas aéreas de Baccharis coridifolia DC. (Asteraceae-Astereae). Lat. Am. J. Pharm. 26: 723-31.

CASTRO, H., V CASALI, L BARBOSA. \& P. CECON. 1999. Rendimiento de tanino em dois acessos de Carqueja (Baccharis myriocephala D.C.), em diferentes épocas de colheita em vicosa-MG. Rev. Bras. Plantas Med. 1: 29-33.

CORDATI, A. \& M. GATTUSO. 1994. Caracterización anatómica e histoquímica de Eupatorium macrocephalum Less., E. inulaefolum H.B.K. y E. subhastatum Hook et Arn. (Asteraceae). Dominguezia 11: 32-42.

D' AMBROGIO DE ARGUESO, A. 1986. Manual de Técnicas de Histología Vegetal. Pp. Ed. Hemisferio Sur. Buenos Aires.

DELBÓN, N., M. CORTEZ, L. CASTELlO, A. RIOS, M. RISSO, N. DOTTORI \& M. T. COSA. 2010. Anatomía foliar y estrategias adaptativas en especies arbustivas de las Sierras Chicas de Córdoba, Argentina. Arnaldoa 17: 41-49.

DELBÓN, N., M. T. COSA. \& G. BERNARDELlO. 2012. Exomorfología y anatomía de órganos vegetativos aéreos en especies de Flourensia DC. (Asteraceae) con importancia fitoquímica. Acta Bot. Bras. 26: 2-10.

DI RIENZO, J., F. CASANOVES, M. BALZARINI, L. GONZALEZ, M. TABLADA \& C. ROBLEDO. InfoStat, versión 2011. Grupo InfoStat. Universidad Nacional de Córdoba.. Córdoba.

ESPÍRITO-SANTO, M., G. FERNANDES, L. ALLAIN. \& T. REIS. 1999. Tannins in Baccharis dracunculifolia D.C. (Asteraceae): effects of seasonality, water availability and plant sex. Acta Bot. Bras. 13: 167-174.
FAHN, A. \& D. CUTLER. 1992. Xerophytes. Handbuchder Pflanzenantomie XIII, 3. Gebruder Borntraeger, Berlín.

FAVIER, L., C. TONN, E. GUERREIRO, A. ROTELLI \& L. PELZER. 1998. Anti-inflammatory activity of acetophenones from Ophryosporus axilliflorus, Plantas Medicinales 64: 657-659.

FEENY, P. P. 1970. Seasonal changes in oak leaf tannins and nutrients as a cause of spring feeding by winter moth caterpillars. Ecology 51: 565-581.

FREIRE, S. \& E. URTUBEY. 1999. Compuestas medicinales de la Provincia Biogeográfica Pampeana: Claves para su determinación e iconografía. Parte II: Compuestas con capítulos isomorfos y papus no piloso o ausente (Grupo 3), Acta Farm. Bonaer. 18: 283-294.

FREIRE, S. E., E. URTUBEY \& D. A. GIULIANO. 2007. Epidermal characters of Baccharis (Asteraceae) species used in traditional medicine. Caldasia 29: 23-38.

GIORGIS, M., A. CINGOLANI, J. CHIARINI, J. CHIAPELLA, G. BARBOZA, L. ARIZA ESPINAR, R. MORERO, D. GURVICH, P. TECCO, R. SUBILS \& M. CABIDO. 2011. Composición florística del Bosque Chaqueño Serrano de la provincia de Córdoba, Argentina. Kurtziana 36: 9-43.

GIORGIS, M., A. M. CINGOLANI \& M. CABIDO. 2013. El efecto del fuego y las características topográficas sobre la vegetación y las propiedades del suelo en la zona de transición entre bosques y pastizales de las sierras de Córdoba, Argentina. Bol. Soc. Argent. Bot. 48: 493-513.

GURVICH, D. E., L. ENRICO \& A. M. CINGOLANI. 2005. Linking plant functional traits with postfire sprouting vigour in woody species in central Argentina. Austral Ecol. 30: 789-796.

HOYOS, L. E., G. I. GAVIER-PIZARRO, T. KUEMMERLE, E. H. BUCHER, V. C. RADELOFF \& P. A. TECCO. 2010. Invasion of glossy privet (Ligustrum lucidum) and native forest loss in the Sierras Chicas of Córdoba, Argentina. Biol. Invasions 12: 3261-3275.

JAKUPOVIC, J., A. SCHUSTER, U. GANZER, F. BOHLMANN \& P. BOLDT. 1990. Sesqui-and diterpenes from Baccharis species. Phytochemistry 29: 2217-2222.

JOHANSEN, D. 1940. Plant microtechnique. $1^{\circ} \mathrm{ed}$. Mc Gaw Hill Book Company, Inc.

KRAUS, J., H. DE SOUSA, M. REZENDE, N. CASTRO, C. VECCHI \& R. LUQUE. 1998. Astra Blue and Basic Fuchsin double staining of plant materials. Biotech. Histochem. 73: 235-243.

MOLARES, S., S. GONZÁLEZ, A. LADIO \& M. CASTRO. 2009. Etnobotánica, anatomía y caracterización físico-química del aceite esencial 
de Baccharis obovata Hook. et Arn. (Asteraceae: Astereae). Acta Bot. Bras. 23: 578-589.

NUÑEZ, C. \& J. CANTERO. 2000. Las plantas medicinales del sur de la provincia de Córdoba, Fundación Universidad Nacional Río Cuarto, Córdoba.

PÉREZ CUADRA, V \& V. CAMBI. 2014. Morphoanatomical functional traits in xerophytic species of a saline environment. Int. J. Eexper. Bot. 83: 389-396.

RAMAYYA, N. 1962. Studies on de trichomes of some Compositae I. General structure. Bull. Bot. Surv. India 14:177-188.

RODRÍGUEZ, M. A., S. GATTUSO \& M. GATTUSO. 2008. Baccharis crispa y Baccharis trimera (Asteraceae): Revisión y Nuevos Aportes para su Normalización Micrográfica. Lat. Am. J. Pharm.27: 387-397.
STACE, C.A. 1965. Cuticular studies as an aid to plant taxonomy. Bull Brit. Mus. Nat. Hist. 4: 62-63.

VILELA, A., L. GONZÁLEZ-PALEO \& D. RAVETTA. 2011. Metabolismo secundario de plantas leñosas de zonas áridas: mecanismo de producción, función y posibilidades de aprovechamiento. Asoc. Arg. Ecol. 21:317-327.

Recibido el 1 de marzo de 2016, aceptado el 15 de julio de 2016. 